International Journal of Modern Physics E (C) World Scientific Publishing Company

\title{
Effects of transient non-thermal particles on the big bang nucleosynthesis
}

\author{
Tae-Sun Park ${ }^{\dagger} \dagger^{\dagger}$, Kyung Joo Min ${ }^{\ddagger}$ and Seung-Woo Hong ${ }^{\dagger, \ddagger, *}$ \\ $\dagger$ Department of Physics, Sungkyunkwan University \\ Suwon 16419, Korea \\ ${ }^{\dagger \dagger}$ Center for Exotic Nuclei Studies, Institute for Basic Science \\ Daejeon 34126, Korea \\ $\ddagger$ Department of Energy Science, Sungkyunkwan University \\ Suwon 16419, Korea \\ *swhong@skku.ac.kr
}

Received Day Month Year Revised Day Month Year

\begin{abstract}
The effects of introducing a small amount of non-thermal distribution (NTD) of elements in big bang nucleosynthesis (BBN) are studied by allowing a fraction of the NTD to be time-dependent so that it contributes only during a certain period of the BBN evolution. The fraction is modeled as a Gaussian-shaped function of $\log (T)$, where $T$ is the temperature of the cosmos, and thus the function is specified by three parameters; the central temporal position, the width and the magnitude. The change in the average nuclear reaction rates due to the presence of the NTD is assumed to be proportional to the Maxwellian reaction rates but with temperature $T_{\mathrm{NTD}} \equiv \zeta T, \zeta$ being another parameter of our model. By scanning a wide four-dimensional parametric space at about half a million points, we have found about 130 points with $\chi^{2}<1$, at which the predicted primordial abundances of light elements are consistent with the observations. The magnitude parameter $\varepsilon_{0}$ of these points turns out to be scattered over a very wide range from $\varepsilon_{0} \sim 10^{-19}$ to $\sim 10^{-1}$, and the $\zeta$-parameter is found to be strongly correlated with the magnitude parameter $\varepsilon_{0}$. The temperature region with $0.3 \times 10^{9} \mathrm{~K} \lesssim T \lesssim 0.4 \times 10^{9} \mathrm{~K}$ or the temporal region $t \simeq 10^{3}$ s seems to play a central role in lowering $\chi^{2}$.
\end{abstract}

Keywords: Big bang nucleosynthesis; transient; non-thermal distribution

PACS numbers: 26.35.+c, 26.40.+r, 98.80.Ft

\section{Introduction}

Big bang nucleosynthesis (BBN) serves as the standard scenario to address the primordial abundances of light elements of our universe. ${ }^{[16}$ With the baryon-tophoton ratio $\eta_{0}$ determined accurately by the cosmic microwave background radiation measurement of Wilkinson Microwave Anisotropy Probe $e^{7}$ and Planck,$\stackrel{8}{8}$ BBN is basically parameter-free and can explain the abundances of primordial deuteron

${ }^{*}$ Corresponding author 
and ${ }^{4} \mathrm{He}$ quite successfully. However, the BBN prediction for lithium is reported to be about three times bigger than the observation, $9-13$ and has brought a lot of attention (see Refs. 14, 15, for instance).

Incidentally, a puzzling drop in $\mathrm{Li} / \mathrm{H}$ in metal-poor stars has been observed, 16 which imposes a substantial uncertainty on the primordial Li abundance. We refer to, for example, Refs. $17-20$ for stellar models that take into account detailed mechanisms of the Li depletion in those stars. These models, however, do not resolve the Li discrepancy completely 21

There have been theoretical efforts to resolve the above so-called "lithium problem" by altering the assumption that all particles except neutrinos are at thermal equilibrium with the Maxwellian distribution. For example, the in-flight reaction probability has been extensively studied $\sqrt{22} \mid 23$ by taking into account the fact that the particles created from nuclear reactions can have energies in the $\mathrm{MeV}$ range and thus can have a probability of overcoming the Coulomb repulsion to go through nuclear reactions before being thermalized. The effect of such a mechanism turns out to be insufficient to change the abundance significantly. Another interesting approach was attempted by Bertulani et al.,24 where the Maxwell-Boltzmann(MB) distribution was modified to adopt the so-called non-extensive statistics with the results that it only worsens the lithium problem.

Our study is closely related to the work by Kang et al.. ${ }^{25}$ where they have introduced so-called "cosmic rays", which consist of only proton isotopes (protons, deuterons and tritons) that are assumed to have a power-law shaped distribution up to $4 \mathrm{MeV}$. In the study of Kang et al. ${ }^{25}$ the upper limit of the distribution is severely constrained since cosmic rays with energies higher than the $\mathrm{D}(p, n) \mathrm{H}_{2}$ threshold, $3.337 \mathrm{MeV}$, destroy deuterium. By tuning the fraction of the cosmic rays with respect to thermal isotopes of hydrogen to $0.7 \times 10^{-6}$, they could account for the lithium abundance successfully, but with a $5 \%$ reduction of the deuteron abundance. In their approach, the fraction of particles (denoted by $\varepsilon$ ) with nonMaxwellian distribution was treated as time-independent. This assumption may be questioned in that the cosmic rays with such a non-MB distribution may exist only for a certain period rather than being independent of time or temperature.

The question on the origin of the time-dependent cosmic rays is not addressed here, but there are candidates related to, but not limited to, decay or annihilation of relic particles, which would inject cosmic rays to the universe. The outgoing particles of decay processes of relic particles would be rapidly thermalized due to the interactions with background materials, but the decay or annihilation rate will have a non-trivial time-dependence characterized by the lifetimes or the time-dependence of the energies and densities of the relic particles. For example, the decay of the next lightest supersymmetric particle (NLSP) into the lightest supersymmetric particle (LSP) dark matter can produce time-dependent suprathermal particles. Indeed, it was discussed that the stau-NLSP and gravitino-LSP system with stau lifetime $\tau \simeq 10^{3}$ s could resolve the lithium problem with some representative values of the 
model parameters .26 We refer to Ref. 27 and references therein for a comprehensive review on the role of dark matter on the BBN.

In this work, we do not stick to any particular scenario or candidate for the origin of cosmic rays. Instead, we explore the possibility in which the fraction of the NTD is a function of time or temperature. Since temperature is a monotonically decreasing function of time, time dependence can be converted to temperature dependence. The time dependence of the fraction $\varepsilon(T)$ is modeled as a Gaussianshaped function of $\log (T)$ with three parameters for the central temporal position $T_{0}$, the width $\Delta$, and the magnitude $\varepsilon_{0}$ : An explicit functional form will be given in the next section. Although a power-law type distribution would be a reasonable approach, making a realistic model for the non-MB distribution requires an initial condition and the evolution with time. Thus, we make a naive assumption for computational convenience by assuming that the averaged reaction rate $\langle\sigma v\rangle_{i j \rightarrow k l}(T)$ in the presence of the NTD can be approximated as a sum of two Maxwellian reaction rates of particles at temperatures $T$ and $T_{\mathrm{NTD}}$, i.e.,

$$
\langle\sigma v\rangle_{i j \rightarrow k l}(T)=[1-\varepsilon(T)] R_{i j \rightarrow k l}^{t h}(T)+\varepsilon(T) R_{i j \rightarrow k l}^{t h}\left(T_{N T D}\right),
$$

where the subscript " $i j \rightarrow k l$ " is the reaction index, $T_{\mathrm{NTD}}$ stands for "the temperature of the NTD", and $R_{i j \rightarrow k l}^{t h}(T)$ is the usual Maxwell-Boltzmann averaged reaction rate in thermal equilibrium at temperature $T$. We introduce $\zeta \equiv T_{\mathrm{NTD}} / T$ and treat $\zeta$ as a free parameter. Since the non-thermal particles with temperatures less than $T$ would not change the reaction rates significantly, the parameter $\zeta$ is expected to be larger than unity. This assumption of a Maxwell-Boltzmann reaction rate for the NTD simplifies the calculation significantly.

We then scan a wide range of four-dimensional parameter space of $\left(\varepsilon_{0}, T_{0}, \Delta, \zeta\right)$, searching for the best parameters that meet the observational data for the primordial abundances. The details of our model and the calculational method are described in Sec. II, which is followed by the results in Sec. III, and discussions in Sec. IV.

\section{Calculational method}

Let us begin with the discussion of the assumption in Eq. (1). The averaged reaction rate of the reaction $i+j \rightarrow k+l$ reads

$$
\begin{array}{r}
\langle\sigma v\rangle_{i j \rightarrow k l}(T)=\frac{1}{2} \int_{-1}^{1} d \cos \theta_{i j} \int_{0}^{\infty} d E_{i} \int_{0}^{\infty} d E_{j} \\
v_{i j} \sigma_{i j \rightarrow k l} f_{i}\left(E_{i}, T\right) f_{j}\left(E_{j}, T\right),
\end{array}
$$

where $\theta_{i j}$ is the relative angle, $v_{i j}$ is the relative velocity, $\sigma_{i j \rightarrow k l}$ is the cross section of the reaction, and $f_{i}\left(E_{i}, T\right)$ is the distribution of the $i$-th particle with energy $E_{i}$. Equation (2) can be rewritten as an integration over the center-of-mass energy $E_{i j}$,

$$
\langle\sigma v\rangle_{i j \rightarrow k l}(T)=\int_{0}^{\infty} d E_{i j} v_{i j} \sigma_{i j \rightarrow k l} F_{i j}\left(E_{i j}, T\right) .
$$


When all the incoming particles are in thermal equilibrium with Maxwellian distribution, $f_{i}\left(E_{i}, T\right)=f^{t h}\left(E_{i}, T\right) \equiv 2 \sqrt{E_{i} / \pi(k T)^{3}} e^{-E_{i} / k T}, F_{i j}\left(E_{i j}, T\right)$ becomes identical to the Maxwell-Boltzmann distribution, $F_{i j}\left(E_{i j}, T\right)=f^{t h}\left(E_{i j}, T\right)$. In the presence of non-thermal components, the $i$-th particle's distribution can be written as

$$
f_{i}\left(E_{i}, T\right)=\left[1-\varepsilon_{i}(T)\right] f^{t h}\left(E_{i}, T\right)+\varepsilon_{i}(T) f_{i}^{n t d}\left(E_{i}, T\right)
$$

with the normalization condition $\int_{0}^{\infty} d E_{i} f_{i}^{n t d}\left(E_{i}, T\right)=1$, where $f_{i}^{n t d}\left(E_{i}, T\right)$ is a yet unknown distribution of the non-thermal part, and $\varepsilon_{i}(T)$ is its temperaturedependent magnitude. Then $F_{i j}$ reads in non-relativistic limit

$$
\begin{aligned}
F_{i j}\left(E_{i j}, T\right)= & \left(1-\varepsilon_{i}\right)\left(1-\varepsilon_{j}\right) f^{t h}\left(E_{i j}, T\right) \\
& +\int_{0}^{\infty} d E_{i} \int_{E_{j-}}^{E_{j+}} d E_{j} \frac{\mathcal{F}_{i j}^{n t d}}{2 \mu v_{i} v_{j}}
\end{aligned}
$$

with

$$
\begin{aligned}
\mathcal{F}_{i j}^{n t d}= & \varepsilon_{i}\left(1-\varepsilon_{j}\right) f_{i}^{n t d}\left(E_{i}, T\right) f^{t h}\left(E_{j}, T\right) \\
& +\left(1-\varepsilon_{i}\right) \varepsilon_{j} f^{t h}\left(E_{i}, T\right) f_{j}^{n t d}\left(E_{j}, T\right) \\
& +\varepsilon_{i} \varepsilon_{j} f_{i}^{n t d}\left(E_{i}, T\right) f_{j}^{n t d}\left(E_{j}, T\right),
\end{aligned}
$$

where $\varepsilon_{i}=\varepsilon_{i}(T), \mu=m_{i} m_{j} /\left(m_{i}+m_{j}\right), v_{i j}=\sqrt{2 E_{i j} / \mu}, v_{i}=\sqrt{2 E_{i} / m_{i}}$, and $E_{j \pm}=\frac{1}{2} m_{j}\left(v_{i j} \pm v_{i}\right)^{2}$. Instead of modeling $f_{i}^{n t d}$, we assume that $F_{i j}\left(E_{i j}, T\right)$ may be effectively approximated as

$$
F_{i j}\left(E_{i j}, T\right)=[1-\varepsilon(T)] f^{t h}\left(E_{i j}, T\right)+\varepsilon(T) f^{t h}\left(E_{i j}, T_{\mathrm{NTD}}\right),
$$

where $\varepsilon(T)$ is the amount of the NTD to be taken as $T$-dependent and is to be discussed shortly. Combining Eqs.(3) and (7), and defining $R_{i j \rightarrow k l}^{t h}(T)$ by

$$
R_{i j \rightarrow k l}^{t h}(T) \equiv \int_{0}^{\infty} d E_{i j} v_{i j} \sigma_{i j \rightarrow k l} f^{t h}\left(E_{i j}, T\right),
$$

we are led to Eq. (1). One of the immediate advantages in this approach is that we can make use of the well-established codes $28+36$ available for the standard BBN calculations without having to write extra codes for the averaged reaction rates in the presence of NTD particles.

Before going further, let us remark on the reverse rates. In the presence of NTD, our assumption given in Eq. (1) implies the inverse rate $k l \rightarrow i j$ to be

$$
\langle\sigma v\rangle_{k l \rightarrow i j}(T)=[1-\varepsilon(T)] R_{k l \rightarrow i j}^{t h}(T)+\varepsilon(T) R_{k l \rightarrow i j}^{t h}\left(T_{N T D}\right) .
$$

As is the case with $R_{k l \rightarrow i j}^{t h}(T)$, the reverse rate $R_{k l \rightarrow i j}^{t h}\left(T_{N T D}\right)$ is computed from $R_{i j \rightarrow k l}^{t h}\left(T_{N T D}\right)$ by using the time reversal symmetry with the assumption of thermal equilibrium at temperature $T_{N T D}$. Because both $R_{i j \rightarrow k l}^{t h}(T)$ and $R_{i j \rightarrow k l}^{t h}\left(T_{N T D}\right)$ satisfy the principle of the detailed balance, the total reverse rate also does. 


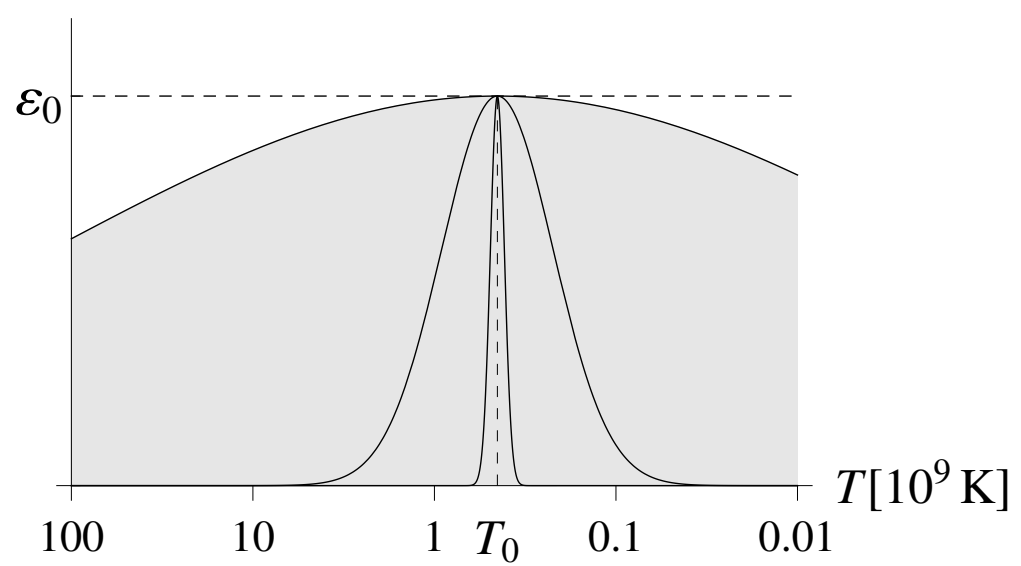

Fig. 1: The NTD portion $\varepsilon(T)$ for $T_{0}=0.45 \times 10^{9} \mathrm{~K}$, and $\Delta=4$ (outermost), 1 (middle) and 0.0625 (innermost).

In the consideration of the time or temperature dependence of the amount of NTD portion, the dependence of $\varepsilon(T)$ on $T$ is taken as a Gaussian-shaped function peaked at $T_{0}$ with a "window" of width $\Delta$ and peak height $\varepsilon_{0}$,

$$
\varepsilon(T)=\varepsilon_{0} \exp \left[-\left(\frac{\log \left(T / T_{0}\right)}{\Delta}\right)^{2}\right]
$$

Note that $\varepsilon(T) / \varepsilon_{0} \geq 1 / e$ only in the region $T_{0} e^{-\Delta} \leq T \leq T_{0} e^{\Delta}$. To illustrate the dependence of $\varepsilon(T)$ on $\Delta$, which corresponds to the length of time during which NTD particles appear, we show $\varepsilon(T)$ for $T_{0}=0.45 \times 10^{9} \mathrm{~K}$ and three values of $\Delta$ in Fig. 1. Since the NTD portion is concentrated around $T \simeq T_{0}, T_{\mathrm{NTD}}=\zeta T$ may be regarded as $T_{\mathrm{NTD}} \simeq \zeta T_{0}$, especially when the width parameter $\Delta$ is small.

Here we would like to mention that our choice for the shape of the fraction $\varepsilon(T)$ as a Gaussian is a naive ansatz chosen for computational convenience. An exponentially decaying shape, for example, could be a more suitable choice for many scenarios of the NTD. However, since we will scan the parametric space with varying the width of $\varepsilon(T)$, the major effect due to the presence of the NTD particle may be captured regardless of the details of the functional form.

Let us now describe how the parameter space is scanned. For the magnitude parameter $\varepsilon_{0}$, we scan quite a large range by choosing $n=1$ to 30 in $\varepsilon_{0}=10^{-n}$ to see the possibility that even a very tiny fraction of NTD may affect the lithium problem. Most important nuclear synthesis occurs around $T \sim(0.1 \sim 1) \times 10^{9} \mathrm{~K}$, and thus for the peak temperature parameter $T_{0}$ we choose 41 grid points in the $\log \left(T_{0}\right)$ axis from $T_{09} \equiv T_{0} / 10^{9} \mathrm{~K}=0.1$ to 1 by setting $T_{09}=10^{n / 40}$ with $n=-40$ to 0 . The width parameter $\Delta$ is chosen to vary from 0.0625 to 4 by doubling the values 
Authors' Names

Table 1: Particle Data Group data and the SBBN prediction for the primordial abundances.

\begin{tabular}{lllll|l}
\hline & $\mathrm{D} / \mathrm{H}\left[10^{-5}\right]$ & $Y_{\mathrm{p}}$ & $\mathrm{Li} / \mathrm{H}\left[10^{-10}\right]$ & \multicolumn{1}{|l}{ Reference } \\
\hline PDG (2012) & $2.82 \pm 0.21$ & $0.249 \pm 0.009$ & $1.7 \pm 0.06 \pm 0.44$ & 37 \\
PDG (2014) & $2.53 \pm 0.04$ & $0.2465 \pm 0.0097$ & $1.6 \pm 0.3$ & $\overline{38}$ \\
SBBN & $2.49 \pm 0.17$ & $0.2486 \pm 0.0002$ & $5.24_{-0.62}^{+0.71}$ & 39 \\
\hline
\end{tabular}

of $\Delta$, that is, $\Delta=2^{n}$ with $n=-4$ to 2 . As shown in Fig. 1] $\Delta=0.0625$ is narrow enough to explore the possibility of locating the period of time for non-thermal disturbance which can affect the BBN results, and $\Delta=4$ is big enough for $\varepsilon(T)$ to be essentially regarded as independent of $T$. The ratio of the NTD-temperature to the temperature of the cosmos, $\zeta=T_{\mathrm{NTD}} / T$, is chosen to be $\zeta=10^{n / 40}$ with $n \geq 1$. Since the Kawano code is supposed to be accurate only up to $T_{9} \equiv T / 10^{9} \mathrm{~K} \lesssim 10$ and the reliability of the calculation for $\zeta T_{09} \gtrsim 10$ is highly questionable, we set an upper limit to the $\zeta$ parameter by imposing the condition $\zeta T_{09} \leq 10$, i.e., $n \leq 40$ for $T_{09}=1$ and $n \leq 80$ for $T_{09}=0.1$. In this search scheme, the total number of grid points considered is $30 \times 41 \times 7 \times(40+80) / 2=516,600$, which can be summarized as follows:

$$
\begin{aligned}
\varepsilon_{0} & =\left[10^{-1}, 10^{-2}, 10^{-3}, \cdots, 10^{-30}\right], \\
T_{09} & =\left[10^{-1}, 10^{-0.975}, 10^{-0.95}, \cdots, 10^{0}\right], \\
\Delta & =[0.0625,0.125,0.25, \cdots, 4], \\
\zeta & =\left[10^{0.025}, 10^{0.05}, 10^{0.075}, \cdots, 10 / T_{09}\right] .
\end{aligned}
$$

At each grid point, we evaluate $\chi^{2}$ defined by

$$
\chi^{2}=\left|\chi\left(\mathrm{D} /\left.\mathrm{H}\right|_{p}\right)\right|^{2}+\left|\chi\left(Y_{\mathrm{p}}\right)\right|^{2}+\left|\chi\left(\mathrm{Li} /\left.\mathrm{H}\right|_{p}\right)\right|^{2}
$$

with

$$
\chi(\alpha) \equiv \frac{\mathcal{C}(\alpha)-\mathcal{O}(\alpha)}{\sigma(\alpha)},
$$

where $\mathcal{C}(\alpha), \mathcal{O}(\alpha)$ and $\sigma(\alpha)$ stand for the calculated value, the observed value, and the uncertainty for the quantity $\alpha$, respectively. For $\mathcal{O}(\alpha)$ and $\sigma(\alpha)$, we adopt the PDG(2014) data listed in Table1.

Here and hereafter, what we mean by $\mathrm{Li}$ in Eq. 12 is the sum of ${ }^{6} \mathrm{Li},{ }^{7} \mathrm{Li}$ and ${ }^{7} \mathrm{Be}$. This is because all the primordial ${ }^{7} \mathrm{Be}$ decays to ${ }^{7} \mathrm{Li}$, and what is measured is the sum of ${ }^{6} \mathrm{Li}$ and ${ }^{7} \mathrm{Li}$, not just ${ }^{7} \mathrm{Li}$, though the amount of primordial ${ }^{6} \mathrm{Li}$ is orders of magnitude less than that of ${ }^{7} \mathrm{Li}$.

For the calculation, we adopt the so-called Kawano code. 28 


\section{Results}

\subsection{Distribution of $\chi^{2}$ in the parameter space}

The results of our calculations of $\chi^{2}$ are presented in the three-dimensional parameter space $\left(\zeta, \varepsilon_{0}, T_{09}\right)$ in Fig. 2 for a few selected values of $\Delta$ by drawing rectangular boxes whose sizes are proportional to the value of $\exp \left(-\chi^{2} / 3\right)$ at each grid site for the cases when the value of $\chi^{2} \leq 3$. The projections of the rectangular boxes are also shown on the three planes in the parameter space $\left(\zeta, \varepsilon_{0}, T_{09}\right)$.

Figure 2(a) shows that when the width parameter is as small as $\Delta=0.0625$, the parameter set with a small $\chi^{2}$ are located in a narrow temperature range with $0.37 \lesssim T_{09} \lesssim 0.43$. This band structure becomes irregular as $\Delta$ becomes bigger, and then disappears for $\Delta \geq 1$. We may understand this result as follows. Equation 10 implies that the magnitude of the non-thermal distribution $\varepsilon(T)$ is non-trivial only during the period when the temperature lies in $T \simeq T_{0}\left(e^{-\Delta} \sim e^{\Delta}\right)$; otherwise the magnitude is exponentially suppressed. If $\Delta$ is small, the NTD is effective only in a narrow range around $T \simeq T_{0}$, and thus the $\chi^{2}$ minimum can be localized in the $T_{0}$ space. The results with a small $\Delta$ in Fig. 2 imply that the NTD contribution in the narrow range around $T \simeq 0.40 \times 10^{9} \mathrm{~K}$ plays the key role in lowering $\chi^{2}$. On the other hand, if $\Delta$ is large, $\varepsilon(T)$ becomes less sensitive to the $T_{0}$ parameter. As long as the "window" of $\varepsilon(T)$ overlaps to some extent with the temperature range around $T \simeq 0.40 \times 10^{9} \mathrm{~K}$, there is a potential to cure the lithium problem. The estimation of the range of $T_{09}$ by using $T_{09} \simeq 0.4 \times\left(e^{-\Delta} \sim e^{\Delta}\right)$, inferred from Eq. 10 gives us $T_{09} \simeq(0.38 \sim 0.43)$ for $\Delta=0.0625, T_{09} \simeq(0.31 \sim 0.51)$ for $\Delta=0.25$ and $T_{09} \simeq(0.15 \sim 1)$ for $\Delta=1$, which are more or less consistent with Fig. 2 . Fig. 2 (d) shows there are not many grid points where $\chi^{2} \leq 3$ when $\Delta=4$. As will be shown in subsection 3.2 , we cannot find good parameter sets with $\chi^{2} \leq 3$ if $\Delta=4$. Thus, the width parameter $\Delta$ seems to be limited to $\Delta=1$ or less.

As can be seen from the projections plotted on the bottom plane in Fig. 2 , the parameter $\zeta=T_{\mathrm{NTD}} / T$ turns out to be strongly correlated with $\varepsilon_{0}$. This is natural because the amount of the NTD portion required to cure the lithium problem would be smaller if the temperature of the NTD becomes higher. It is noteworthy that the correlation curves are quite insensitive to the value of $\Delta$, which can be seen by comparing the projections in the $\left(\varepsilon_{0}, \zeta\right)$ plane for different values of $\Delta$ in Fig. 2 , The projection of $\exp \left(-\chi^{2} / 3\right)$ on the $\left(\varepsilon_{0}, \zeta\right)$ plane is displayed in Fig. 3 , where the value of $\Delta$ is adjusted to yield the minimum $\chi^{2}$ at each grid site. If $\zeta$ is chosen as 20, 10 and 5, the required fraction to yield a small $\chi^{2}$ becomes $\varepsilon_{0} \simeq 10^{-17}, 10^{-15}$ and $10^{-11}$, respectively.

In Fig. 4. $\exp \left(-\chi^{2} / 3\right)$ is plotted in the $\left(\varepsilon_{0}, T_{09}\right)$ plane for a few selected values of $\Delta$. At each grid point, the value of $\zeta$ is further adjusted to produce the minimum

$\chi^{2}$ while its rough value can be inferred from the aforementioned correlation curve between $\varepsilon_{0}$ and $\zeta$. Figure 4 demonstrates, in particular, how the band-type structure observed around $T_{09} \simeq 0.40$ for a small $\Delta$ evolves as $\Delta$ becomes large. For a width parameter $\Delta$ in the medium range, such as $\Delta=0.25$, the band is formed in the 


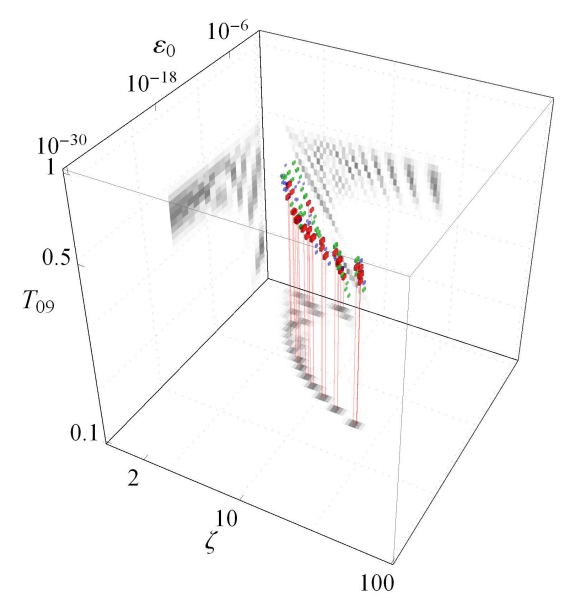

(a) $\Delta=0.0625$

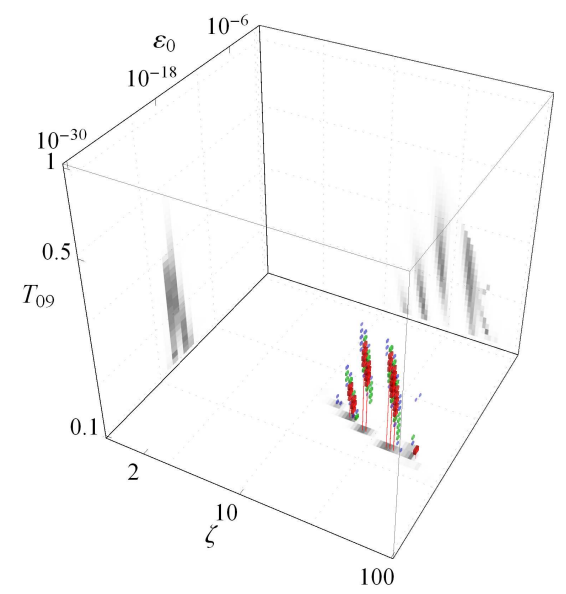

(c) $\Delta=1$

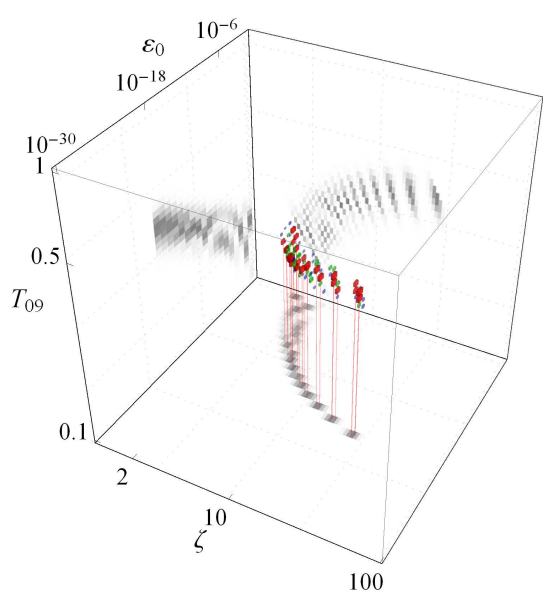

(b) $\Delta=0.25$

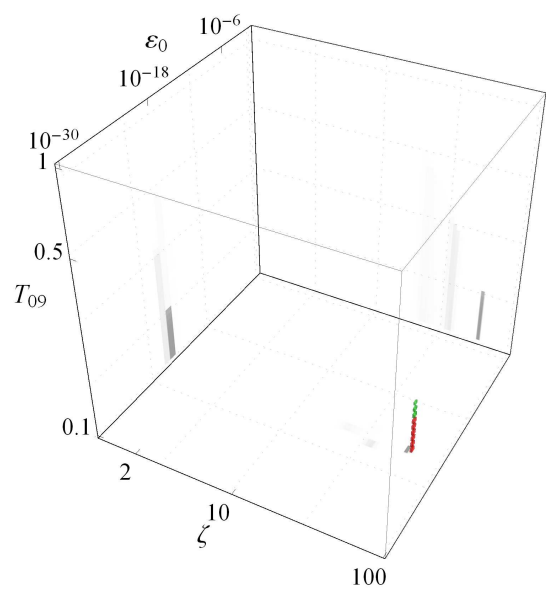

(d) $\Delta=4$

Fig. 2: The $\chi^{2}$ values are plotted in the three-dimensional parameter space $\left(\varepsilon_{0}, \zeta, T_{09}\right)$ for $\Delta=0.0625$ (a), 0.25 (b), 1 (c) and 4 (d). Though the grids are not shown here, the grid points at which $\chi^{2} \leq 3$ are represented by rectangular boxes, whose sizes are proportional to the value of $e^{-\chi^{2} / 3}$. The boxes are colored according to the value of $\chi^{2}$ : Red for $\chi^{2} \leq 1$, green for $1<\chi^{2} \leq 2$ and blue for $2<\chi^{2} \leq 3$. For the cases where $\chi^{2} \leq 1$, thin lines are drawn to guide the eyes from the boxes down to the bottom plane. The boxes are projected to the three planes $\left(\varepsilon_{0}, \zeta\right),\left(\zeta, T_{0}\right)$, and $\left(T_{0}, \varepsilon_{0}\right)$, where a darker gray color corresponds to a smaller $\chi^{2}$.

diagonal direction in the $\left(\varepsilon_{0}, T_{09}\right)$ plane, while the band is rotated to the direction of constant $\varepsilon_{0} \sim 10^{-18}$ for $\Delta=1$ or larger. 
Table 2: 12 points in the parametric space which have $\chi^{2}<0.1$. To get the values of $\chi^{2}$ per degree of freedom, $1 / 3$ needs to be multiplied by the values in the Table.

\begin{tabular}{rlllllllllll}
\hline No & $\chi^{2}$ & $\varepsilon_{0}$ & $\Delta$ & $\zeta$ & $\begin{array}{l}T_{09} \\
{\left[10^{9} \mathrm{~K}\right]}\end{array}$ & $\begin{array}{l}\mathrm{D} / \mathrm{H} \\
{\left[10^{-5}\right]}\end{array}$ & $\begin{array}{l}\mathrm{T} / \mathrm{H} \\
{\left[10^{-8}\right]}\end{array}$ & $\begin{array}{l}{ }^{3} \mathrm{He} / \mathrm{H} \\
{\left[10^{-5}\right]}\end{array}$ & $\begin{array}{l}{ }^{6} \mathrm{Li} / \mathrm{H} \\
{\left[10^{-14}\right]}\end{array}$ & $\begin{array}{l}{ }^{7} \mathrm{Li} / \mathrm{H} \\
{\left[10^{-10}\right]}\end{array}$ & $\begin{array}{c}{ }^{7} \mathrm{Be} / \mathrm{H} \\
{\left[10^{-10}\right]}\end{array}$ \\
\hline 1 & 0.006 & $10^{-12}$ & 0.2500 & 5.62 & 0.282 & 2.528 & 5.692 & 0.844 & 1.091 & 0.242 & 1.355 \\
2 & 0.011 & $10^{-15}$ & 0.5000 & 10.6 & 0.200 & 2.532 & 6.103 & 0.834 & 1.093 & 0.331 & 1.291 \\
3 & 0.015 & $10^{-19}$ & 1.0000 & 42.2 & 0.188 & 2.532 & 10.60 & 0.936 & 1.099 & 0.527 & 1.045 \\
4 & 0.032 & $10^{-16}$ & 0.1250 & 12.6 & 0.398 & 2.535 & 5.638 & 0.838 & 1.095 & 0.189 & 1.376 \\
5 & 0.046 & $10^{-12}$ & 0.0625 & 5.31 & 0.398 & 2.528 & 5.623 & 0.841 & 1.091 & 0.181 & 1.358 \\
6 & 0.048 & $10^{-02}$ & 0.1250 & 2.82 & 0.224 & 2.535 & 6.482 & 0.956 & 1.095 & 0.458 & 1.093 \\
7 & 0.054 & $10^{-01}$ & 0.0625 & 4.47 & 0.141 & 2.527 & 14.40 & 1.021 & 1.195 & 0.402 & 1.262 \\
8 & 0.063 & $10^{-11}$ & 0.2500 & 5.01 & 0.266 & 2.522 & 5.683 & 0.842 & 1.089 & 0.246 & 1.315 \\
9 & 0.063 & $10^{-17}$ & 1.0000 & 20.0 & 0.112 & 2.521 & 7.534 & 0.823 & 1.089 & 0.434 & 1.186 \\
10 & 0.064 & $10^{-19}$ & 1.0000 & 42.2 & 0.178 & 2.528 & 10.65 & 0.942 & 1.098 & 0.537 & 1.135 \\
11 & 0.069 & $10^{-01}$ & 0.1250 & 2.11 & 0.282 & 2.539 & 5.821 & 0.950 & 1.097 & 0.188 & 1.379 \\
12 & 0.077 & $10^{-16}$ & 0.5000 & 13.3 & 0.224 & 2.521 & 6.273 & 0.832 & 1.088 & 0.361 & 1.282 \\
\hline
\end{tabular}

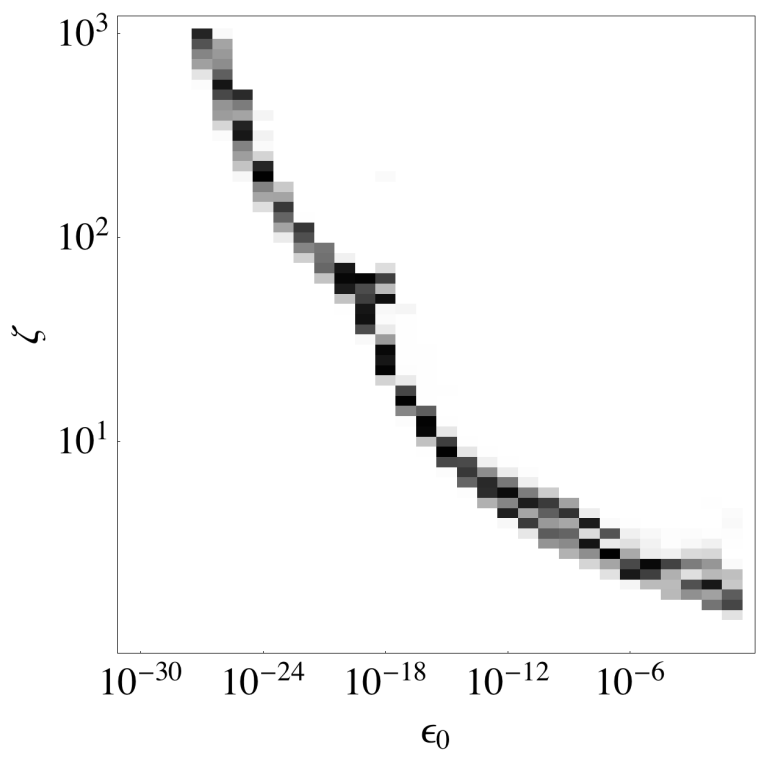

Fig. 3: Projection of $\exp \left(-\chi^{2} / 3\right)$ on the $\left(\varepsilon_{0}, \zeta\right)$ plane, while the value of $\Delta$ is adjusted to yield the minimum $\chi^{2}$ at each grid site.

\subsection{Parameter sets with small values of $\chi^{2}$}

More than 130 grid points are found to yield $\chi^{2}$ less than 1 . Among them, 12 cases have $\chi^{2}<0.1$, which are listed in Table 2 with the resulting abundances of light 
Table 3: The parameter sets with minimum $\chi^{2}$ for each value of $\varepsilon_{0}$. Only the cases with $\chi^{2}<10$ are listed.

\begin{tabular}{lcccccccccc}
\hline \hline$\varepsilon_{0}$ & $\Delta$ & $\zeta$ & $\begin{array}{c}T_{09} \\
{\left[10^{9} \mathrm{~K}\right]}\end{array}$ & $\chi^{2}$ & $\begin{array}{l}\mathrm{D} / \mathrm{H} \\
{\left[10^{-5}\right]}\end{array}$ & $\begin{array}{l}\mathrm{T} / \mathrm{H} \\
{\left[10^{-8}\right]}\end{array}$ & $\begin{array}{l}{ }^{3} \mathrm{He} / \mathrm{H} \\
{\left[10^{-5}\right]}\end{array}$ & $\begin{array}{l}{ }^{6} \mathrm{Li} / \mathrm{H} \\
{\left[10^{-14}\right]}\end{array}$ & $\begin{array}{l}{ }^{7} \mathrm{Li} / \mathrm{H} \\
{\left[10^{-10}\right]}\end{array}$ & ${ }^{7} \mathrm{Be} / \mathrm{H}$ \\
{$\left[10^{-10}\right]$}
\end{tabular}

elements. The number of minima itself is not meaningful since it will become larger if we take a finer mesh. In Table 3 , the values of $\Delta, \zeta$ and $T_{09}$ which minimize $\chi^{2}$ for each value of $\varepsilon_{0}$ considered here are presented together with the abundances of elements. There is no parameter set resulting in $\chi^{2}<10$ for $\varepsilon_{0} \leq 10^{-21}$, and for $\varepsilon_{0} \geq 10^{-19}$ the parameter sets with $\chi^{2}<1$ are found.

In all the cases listed, the proton and the ${ }^{4} \mathrm{He}$ abundances are found to be 0.753 and 0.247 , respectively. The abundance of ${ }^{6} \mathrm{Li} / \mathrm{H}$ is quite insensitive to the value of $\varepsilon_{0}$, resulting in ${ }^{6} \mathrm{Li} / \mathrm{H} \simeq 1.1 \times 10^{-14}$. The sum of abundances of ${ }^{7} \mathrm{Li}$ and ${ }^{7} \mathrm{Be}$ is constrained by the $\chi^{2}$ calculation, and is consistent with the measured primordial lithium abundance for all the parameter sets with small $\chi^{2}$ values listed in Table 2 , while there are sizable variations in the abundances of ${ }^{7} \mathrm{Li}$ and ${ }^{7} \mathrm{Be}$.

\subsection{Evolution of abundances}

The evolution of abundance of light elements is shown for two selected cases in Fig. 5 with (the solid lines) and without (the dotted lines) taking into account the NTD contributions. The left panels are for the set No. 1 in Table 2 with $\chi^{2}=0.006$, 


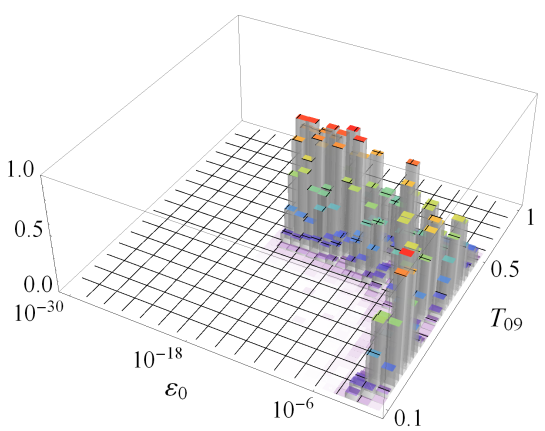

(a) $\Delta=0.0625$

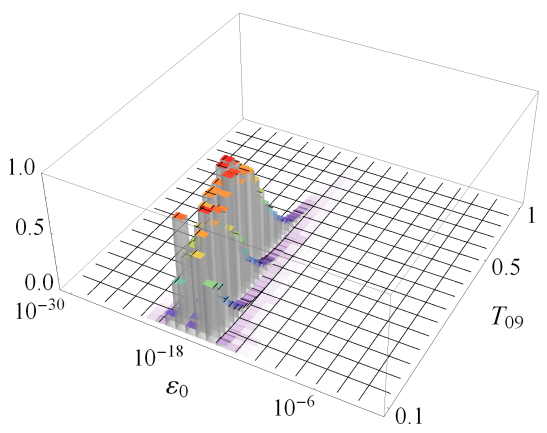

(c) $\Delta=1$

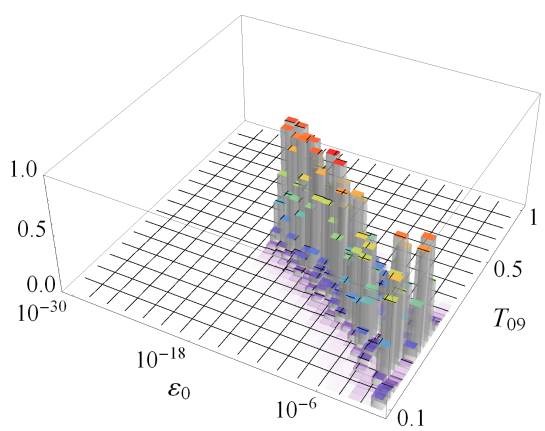

(b) $\Delta=0.25$

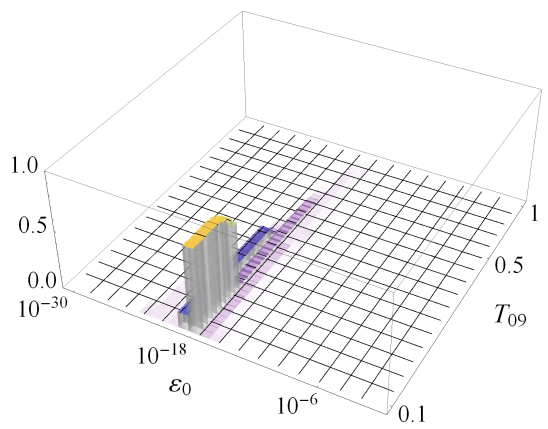

(d) $\Delta=4$

Fig. 4: The value of $\exp \left(-\chi^{2} / 3\right)$ on the $\left(\varepsilon_{0}, T_{09}\right)$ plane, where the width parameter is $\Delta=0.0625$ (a), 0.25 (b), 1 (c) and 4 (d). The value of $\zeta$ is adjusted to yield the minimum $\chi^{2}$ at each grid point.

and the right panels are for the set No. 4 with $\chi^{2}=0.032$. In the bottom panels, the relative ratios of the abundances with and without the NTD particles are plotted.

Several remarks are in order here. First, major changes occur during the period $0.5 \lesssim T_{9} \lesssim 0.3$. Secondly, the NTD contribution is found to lower the abundances of ${ }^{3} \mathrm{He}$ and ${ }^{7} \mathrm{Be}$. The neutron, triton and ${ }^{7} \mathrm{Li}$ abundances increase during this period until $T_{9} \simeq 0.35$ and then slightly decrease. ${ }^{7} \mathrm{Be}$ is dominant over ${ }^{7} \mathrm{Li}$, and the sum of the two is lowered by about one-third, as required by the observation. Thirdly, as the neutron abundance increases around $T_{9}=(0.5 \sim 0.3)$ due to the NTD contribution, Coc et al $\stackrel{40}{ }$ have also concluded that the injection of extra neutrons at $T \simeq 50 \mathrm{keV}$ ( or $T_{9} \simeq 0.43$ ) can resolve the lithium problem but at the cost of over-predicting the deuteron abundance far beyond the observed values.

In Fig. 6, we show the changes in the production rate of light elements due to the NTD contribution

$$
\Delta Y_{i} \equiv \frac{1}{H} \frac{d}{d t}\left[Y_{i}^{n t d}-Y_{i}^{t h}\right]
$$



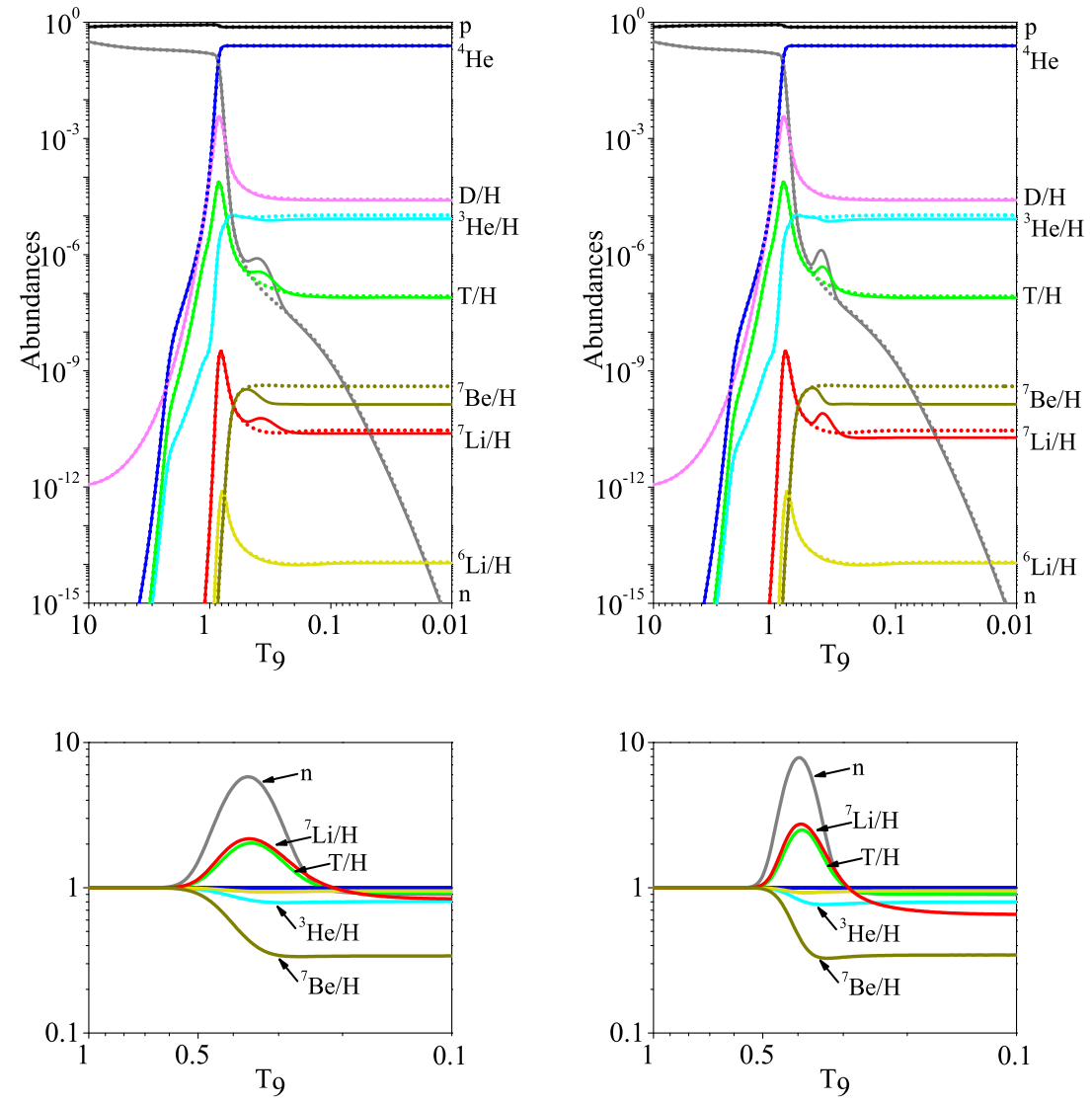

Fig. 5: The abundances of elements with (the solid lines) and without (the dotted lines) the NTD effects are shown in the top panels, and the relative ratios of the abundances of elements with the NTD effects with respect to those without are plotted in the bottom panels. The left panels are for the parameter set No. 1 $\left(\varepsilon_{0}=10^{-12}, \Delta=0.25, \zeta=5.62\right.$ and $\left.T_{0}=0.282\right)$, and the right panels are for the set No. $4\left(\varepsilon_{0}=10^{-16}, \Delta=0.125, \zeta=12.6\right.$ and $\left.T_{0}=0.398\right)$ of Table 2

where $Y_{i}^{\text {ntd }}$ and $Y_{i}^{\text {th }}$ denote the abundance of the neutron $(i=n)$ as shown in Fig. 6(a) and that of the sum of ${ }^{7} \mathrm{Be}$ and ${ }^{7} \mathrm{Li}\left(i={ }^{7} \mathrm{Be}+{ }^{7} \mathrm{Li}\right)$ as shown in Fig. 6(b) with and without the NTD contribution, respectively, and $H$ is the Hubble parameter. The left and right panels are for the parameter sets No. 1 and No. 4 of Table 2 , respectively. In addition to the net change (denoted by the solid lines), we have also plotted the contributions from a few important reactions, i. e., $p+n \rightarrow \mathrm{D}+\gamma$, $\mathrm{T}+\mathrm{D} \rightarrow n+{ }^{4} \mathrm{He},{ }^{3} \mathrm{He}+n \rightarrow p+\mathrm{T},{ }^{7} \mathrm{Li}+p \rightarrow 2{ }^{4} \mathrm{He}$ and ${ }^{4} \mathrm{He}+\mathrm{T} \rightarrow{ }^{7} \mathrm{Li}+\gamma$. The general behaviors of the curves from both sets No. 1 and No. 4 are quite similar. We observe that the NTD component enhances the photo-disintegration of the 
deuteron (the backward reaction of $p+n \rightarrow \mathrm{D}+\gamma$ ) and $\mathrm{T}+\mathrm{D} \rightarrow n+{ }^{4}$ He reaction, increasing the neutron abundance. On the other hand, ${ }^{3} \mathrm{He}+n \rightarrow p+\mathrm{T}$ reaction is also enhanced by the NTD, and reduces the neutron abundance. The bottom panels of Fig. 5 also show the neutron abundance increases until $T_{09} \simeq 0.4$ as much as about 8 times of the SBBN value, and then decreases to the level of 0.9 times of the SBBN value.

The change in ${ }^{7} \mathrm{Li}+{ }^{7} \mathrm{Be}$ abundance is found to be dominated by the NTD contribution from the ${ }^{7} \mathrm{Li}+p \rightarrow 2^{4} \mathrm{He}$ reaction. A high NTD temperature enhances the burning of ${ }^{7} \mathrm{Li}$ by allowing the proton to overcome the Coulomb barrier and to fuse with ${ }^{7} \mathrm{Li}$. Roughly a half of the reduction of Li due to this process is offset by the increase of ${ }^{7} \mathrm{Li}$ abundance through the ${ }^{4} \mathrm{He}+\mathrm{T} \rightarrow{ }^{7} \mathrm{Li}+\gamma$ reaction. Due to the increased neutron abundance, the ${ }^{7} \mathrm{Be}+n \rightarrow p+{ }^{7} \mathrm{Li}$ reaction also increases the lithium abundance roughly as much as ${ }^{4} \mathrm{He}+\mathrm{T} \rightarrow{ }^{7} \mathrm{Li}+\gamma$ reaction, but it lowers the same amount of ${ }^{7} \mathrm{Be}$ leaving the sum of ${ }^{7} \mathrm{Li}+{ }^{7} \mathrm{Be}$ unchanged. After summing the contributions from all the reactions, the lithium abundance is lowered to the measured value.

In Fig. 77 we plotted the abundances of the light elements as functions of the baryon-to-photon ration $\eta$. The solid lines are for the standard BBN without the NTD, and the dotted and dashed lines are for the parameter sets No. 1 and No. 4 , respectively. It shows that the ${ }^{4} \mathrm{He}$ and $\mathrm{D}$ abundances are little changed, while the ${ }^{7} \mathrm{Li}$ abundance is substantially reduced, as required to resolve the "lithium problem". The abundance of ${ }^{3} \mathrm{He} / \mathrm{H}$ is also found to be noticeably reduced by including the NTD. The parameter sets Nos. 1 and 4 give us ${ }^{3} \mathrm{He} / \mathrm{H}=0.85 \times 10^{-5}$ a which is consistent with the upper limit evaluated in Ref., ${ }^{41} \mathrm{He} / \mathrm{H} \leq(1.1 \pm 0.2) \times$ $10^{-5}$. It is to be noted that the primordial abundance of ${ }^{3} \mathrm{He}$ is still uncertain, the only data available coming from the Solar system and solar-matallicity HII regions in the Galaxy ${ }^{41}$ For this reason, we have not included the ${ }^{3} \mathrm{He}$ primordial abundance in our chi-square estimation; see Eq. 12.

\section{Discussions}

We have studied the consequences of introducing a small fraction of non-thermal particles during the BBN process, allowing its magnitude to be time-dependent so that it contributes only for a certain period. This work may be regarded as an extension of the work by Kang et al ${ }^{[25}$ where the magnitude was treated as timeindependent. This extension, however, widens enormously the parametric space to be explored. As the first step in this direction, we have made the assumption of Eq. (1). Therefore, the contributions from the NTD of particles have been modeled in such a way that the average reaction rates are a superposition of two Maxwellian reaction rates of temperature $T$ and $T_{\mathrm{NTD}}=\zeta T$ given by Eq. (1). The calculations are based on the Kawano code, and thus the advances in nuclear cross sections made

\footnotetext{
${ }^{\text {a }}$ What is meant by ${ }^{3} \mathrm{He} / \mathrm{H}$ here is the sum of ${ }^{3} \mathrm{He} / \mathrm{H}$ and $\mathrm{T} / \mathrm{H}$ in Table 2.
} 

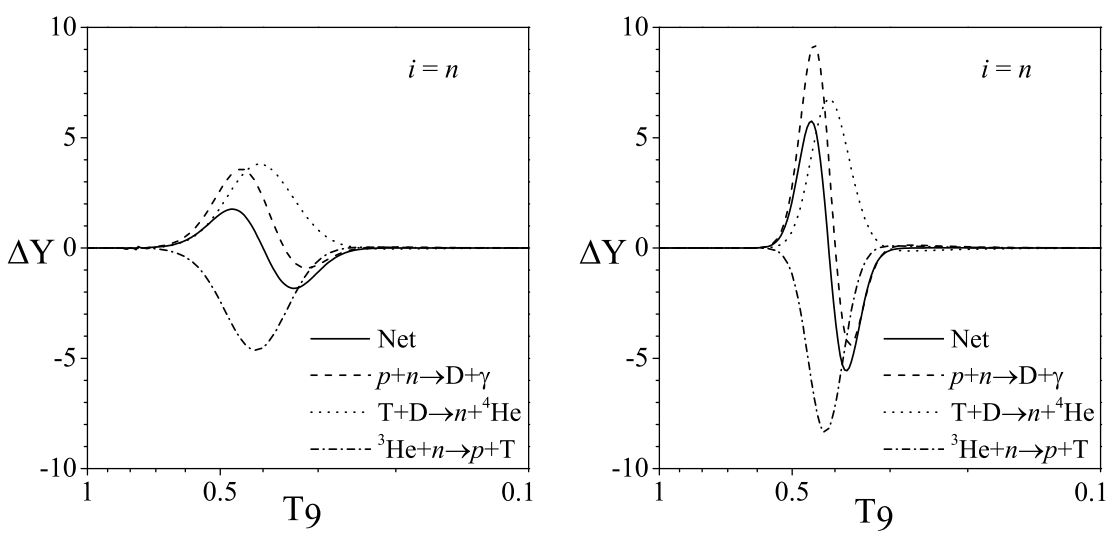

(a) neutron
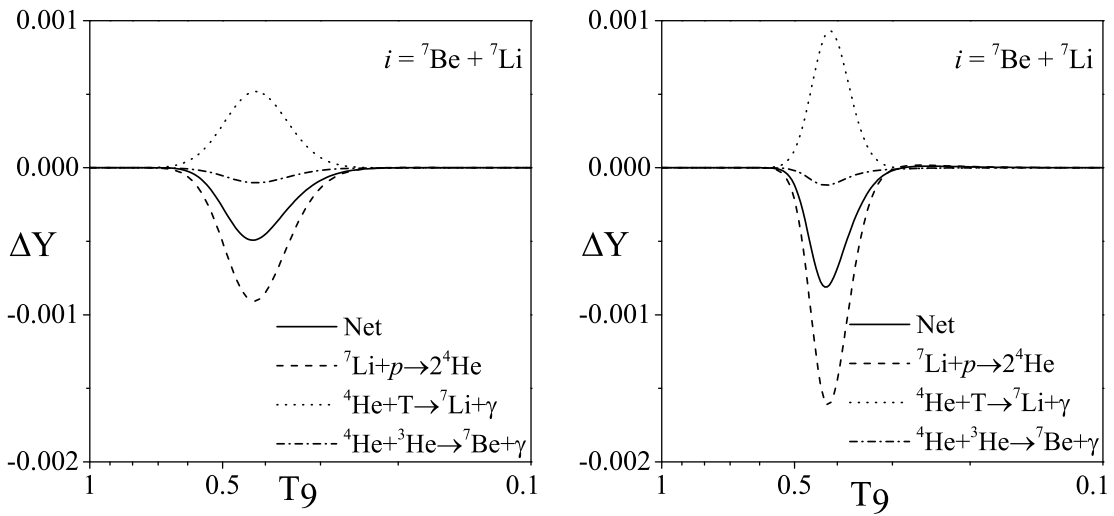

(b) ${ }^{7} \mathrm{Be}+{ }^{7} \mathrm{Li}$

Fig. 6: The NTD induced changes in the production rate, Eq. 14, for neutrons (top) and ${ }^{7} \mathrm{Be}+{ }^{7} \mathrm{Li}$ (bottom). The left and right panels are for the the parameter set No. 1 and No. 4 of Table 2 respectively.

after the establishment of the Kawano code have not been taken into account.

With these caveats mentioned, we are in the position to discuss what is found. By scanning about half a million points in the parameter space, we have found more than 130 points which have $\chi^{2}$ less than 1 . Among them, twelve points have $\chi^{2}<0.1$, in good agreements with the observational data. Those minima are found to be scattered around in the parameter space.

When the width $\Delta$ is small, the parameters with small values of $\chi^{2}$ turn out to be located in a narrow strip in the parametric space around $T_{09} \simeq 0.4$ and a 


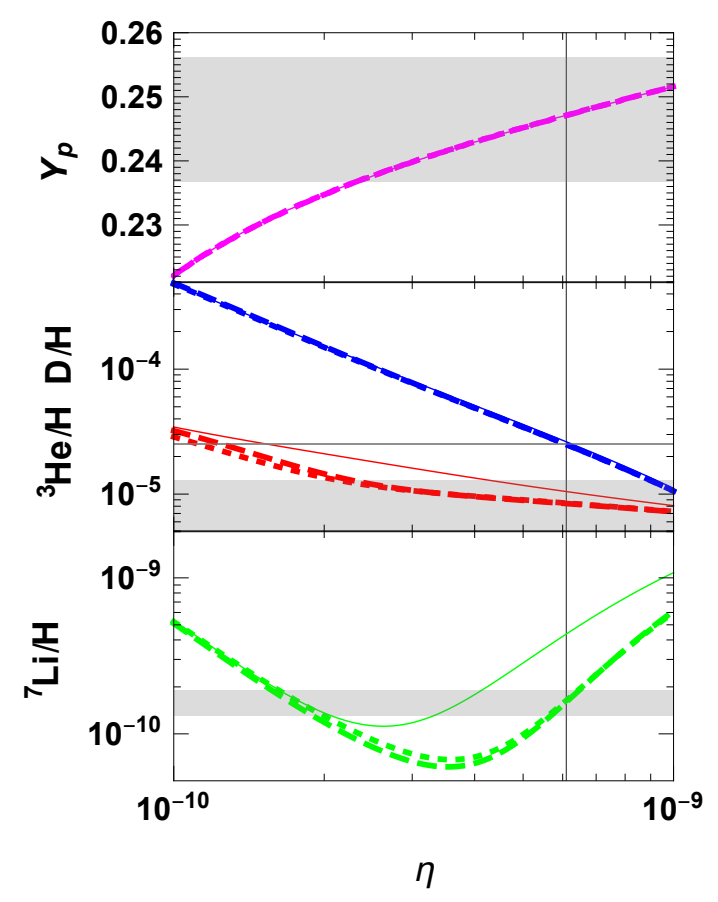

Fig. 7: The primodial abundances with respect to the baryon-to-photon ratio $\eta$. The solid lines are for the standard BBN, and the dotted and dashed lines are for the parameter set No. 1 and No. 4, respectively. The vertical line at $\eta=6.10 \times 10^{-10}$ denotes the value of the ratio $\eta$ obtained by the Planck, $\frac{8}{8}$ and the horizontal bands in gray are the observed light element abundances. ${ }^{38}$

strong correlation between $\varepsilon_{0}$ and $\zeta$ is observed. When the width parameter is as small as 0.0625 , the parameter sets with small $\chi^{2}$ values exist over a range with $0.37 \lesssim T_{09} \lesssim 0.43$. The corresponding temporal range is $1300 \gtrsim t \gtrsim 950$ seconds. Our result is similar to the earlier study, ${ }^{26}$ where it was discussed that the stauNLSP and gravitino-LSP system with stau lifetime $\tau \simeq 10^{3} \mathrm{~s}$ could resolve the lithium problem with some representative values of the model parameters. If the width is as large as $\Delta=1$, we could still find a large number of parameter sets with small $\chi^{2}$ which, however, turns out to be scattered in rather a broad region in the $T_{09}$ parametric space. This may imply that there can be diverse NTD-induced mechanisms that can bring the BBN predictions to the observation data. It is certainly necessary to refine the model to overcome the above mentioned limitations to identify the reaction channels responsible for the cure of the lithium problem.

It would be very useful and interesting to understand what happens when some of the underlying assumptions are released. Furthermore, we have not yet discussed 
"chemical spectrum" of cosmic rays, which will enlarge the parametric space enormously. These extensions are under progress by making use of an updated version for the Kawano code.

\section{Acknowledgements}

We would like to thank Chung Yeol Ryu and Sang-In Bak for valuable discussions. This work was supported by the National Research Foundation of Korea (NRF) funded by the Ministry of Education, Science and Technology (2017R1A2B4012758 and 2013M7A1A1075764). TSP was also partly supported by the Institute for Basic Science (IBS-R031-D1).

\section{References}

1. R. A. Alpher and R. C. Herman, Phys. Rev. 74 (1948) 1737.

2. D. N. Schramm and R. V. Wagoner, Annu. Rev. Nucl. Part. Sci. 27 (1977) 37.

3. P. J. E. Peebles, D. N. Schramm, E. L. Turner and R. G. Kron, Nature 352 (1991) 769.

4. M. S. Smith, L. H. Kawano and R. A. Malaney, Astrophys. J. Suppl. 85 (1993) 219.

5. S. Sarkar, Rep. Prog. Phys. 59 (1996) 1493.

6. G. Steigman, Annu. Rev. Nucl. Part. Sci. 57 (2007) 463.

7. D. N. Spergel et al., Astrophys. J. Suppl. 148 (2003) 175.

8. P. A. R. Ade et al. (Planck Collaboration), Astron. Astrophys. 571 (2014) A16.

9. J. Melendez and I. Ramirez, Astrophys. J. Lett. 615 (2004) L33.

10. M. Asplund, D. L. Lambert, P. E. Nissen, F. Primas and V. V. Smith, Astrophys. J. 664 (2006) 229.

11. M. Kusakabe, T. Kajino and G. J. Mathews, Phys. Rev. 59 (2006) 023526.

12. R. H. Cyburt, B. D. Fields and K. A. Olive, J. Cosmol. Astropart. Phys. 11 (2008) 012.

13. F. Iocco, G. Mangano, G. Miele, O. Pisanti and P. D. Serpico, Phys. Rep. 472 (2009) 1.

14. B. D. Fields, Annu. Rev. Nucl. Part. Sci 61 (2011) 47.

15. G. Israelian, Nature 489 (2012) 37.

16. L. Sbordone et al., Astron. Astrophys. 522 (2010) A26.

17. A. J. Korn et al., Nature 442 (2006) 657.

18. X. Fu, A. Bressan, P. Molaro and P. Marigo, Mon. Not. R. Astron. Soc. 452 (2015) 3256 .

19. M. Spite, F. Spite and P. Bonifacio, Mem. Soc. Astron. Ital. Suppl. 22 (2012) 9.

20. F. Iocco, Mem. Soc. Astron. Ital. Suppl. 22 (2012) 19.

21. M. Tanabashi et al. (Particle Data Group), Phys. Rev. D 98 (2018) 030001.

22. V. T. Voronchev, Y. Nakao and M. Nakamura, Astrophys. J. 725 (2010) 242.

23. V. T. Voronchev, Y. Nakao, K. Tsukida and M. Nakamura, Phys. Rev. D 85 (2012) 067301.

24. C. A. Bertulani, J. Fuqua and M. S. Hussein, Astrophys. J. 767 (2013) 67.

25. M. M. Kang, Y. Hu, H. B. Hu and S. H. Zhu, J. Cosmol. Astropart. Phys. 05 (2012) 011.

26. S. Bailly, K. Jedamzik and G. Moultaka, Phys. Rev. D 80 (2009) 063509.

27. K. Jedamzik and M. Pospelov, New J. Phy. 11 (2009) 105028. 
28. L. Kawano, Let's Go: Early Universe. Guide to Primordial Nucleosynthesis Programming, FERMILAB-PUB-88-34-A (1988).

29. L. Kawano, Let's Go: Early Universe. 2. Primordial nucleosynthesis: The computer way, FERMILAB-PUB-92-004-A (1992).

30. R. V. Wagoner, W. A. Fowler and F. Hoyle, Astrophys. J. 148 (1967) 3.

31. R. V. Wagoner, Astrophys. J. Suppl. 18 (1969) 247.

32. R. V. Wagoner, Astrophys. J. 179 (1973) 343.

33. W. A. Fowler, G. R. Caughlan and B. A. Zimmerman, Annu. Rev. Astron. Astrophys. 5 (1967) 525.

34. W. A. Fowler, G. R. Caughlan and B. A. Zimmerman, Annu. Rev. Astron. Astrophys. 13 (1975) 69

35. M. J. Harris, W. A. Fowler, G. R. Caughlan and B. A. Zimmerman, Annu. Rev. Astron. Astrophys. 21 (1983) 165.

36. G. Beaudet and P. Goret, Astron. Astrophys. 49 (1976) 415.

37. J. Beringer et al. (Particle Data Group) Phys. Rev. D 86 (2012) 010001.

38. K. A. Olive et al. (Particle Data Group) Chin. Phys. C 38 (2014) 090001.

39. R.H. Cyburt, B.D. Fields, K.A. Olive, and T.-H. Yeh, Rev. Mod. Phys. 88 (2016) 015004.

40. A. Coc, M. Pospelov, J. P. Uzan and E. Vangioni, Phys. Rev. D 90 (2014) 085018.

41. T.M. Bania et al., Nat. 415 (2002) 54. 